\title{
Rapid CO gas dispersal from NO Lup's class III circumstellar disc
}

\author{
J. B. Lovell ${ }^{1 \star}$, G. M. Kennedy ${ }^{2,3}$, S. Marino ${ }^{1}$, M. C. Wyatt ${ }^{1}$, M. Ansdell ${ }^{4}$, M. Kama ${ }^{5,1}$, \\ C. F. Manara ${ }^{6}$, L. Matrà ${ }^{7}$, G. Rosotti $^{8}$, M. Tazzari ${ }^{1}$, L. Testi ${ }^{6,9}$, J. P. Williams $^{10}$ \\ ${ }^{1}$ Institute of Astronomy, University of Cambridge, Madingley Road, Cambridge, CB3 OHA, UK \\ ${ }^{2}$ Department of Physics, University of Warwick, Coventry, CV4 7AL, UK \\ ${ }^{3}$ Centre for Exoplanets and Habitability, University of Warwick, Gibbet Hill Road, Coventry CV4 7AL, UK \\ ${ }^{4}$ National Aeronautics and Space Administration Headquarters, 300 E Street SW, Washington DC 20546, USA \\ ${ }^{5}$ Tartu Observatory, University of Tartu, 61602 Töravere, Estonia \\ ${ }^{6}$ European Southern Observatory, Karl-Schwarzschild-Strasse 2, 85748, Garching bei München, Germany \\ ${ }^{7}$ School of Physics, National University of Ireland Galway, University Road, Galway, Ireland \\ ${ }^{8}$ Leiden Observatory, Leiden University, P.O. Box 9513, NL-2300 RA Leiden, the Netherlands \\ ${ }^{9}$ INAF - Osservatorio Astrofisico di Arcetri, L.go E. Fermi 5, 50125, Firenze, Italy \\ ${ }^{10}$ Institute for Astronomy, University of Hawai'i at Mänoa, Honolulu, HI, USA
}

Accepted 2020 November 17. Received 2020 November 17; in original form 2020 October 16

\begin{abstract}
We observed the K7 class III star NO Lup in an ALMA survey of the 1-3 Myr Lupus association and detected circumstellar dust and $\mathrm{CO}$ gas. Here we show that the $\mathrm{J}=3-2 \mathrm{CO}$ emission is both spectrally and spatially resolved, with a broad velocity width $\sim 19 \mathrm{~km} \mathrm{~s}^{-1}$ for its resolved size $\sim 1^{\prime \prime}(\sim 130 \mathrm{au})$. We model the gas emission as a Keplerian disc, finding consistency, but only with a central mass of $\sim 11 M_{\odot}$, which is implausible given its spectral type and $\mathrm{X}$-Shooter spectrum. A good fit to the data can also be found by modelling the $\mathrm{CO}$ emission as outflowing gas with a radial velocity $\sim 22 \mathrm{~km} \mathrm{~s}^{-1}$. We interpret NO Lup's CO emission as the first imaged class III circumstellar disc with outflowing gas. We conclude that the $\mathrm{CO}$ is continually replenished, but cannot say if this is from the break-up of icy planetesimals or from the last remnants of the protoplanetary disc. We suggest further work to explore the origin of this $\mathrm{CO}$, and its higher than expected velocity in comparison to photoevaporative models.
\end{abstract}

Key words: circumstellar matter - planetary systems - submillimetre: planetary systems.

\section{INTRODUCTION}

Stars are born with protoplanetary discs containing large quantities of primordial gas and dust which persist for several Myr before dispersing on rapid $~ 0.1$ Myr timescales (Ercolano \& Pascucci 2017). Circumstellar discs are also seen around older stars ( $\gtrsim 10 \mathrm{Myr})$, known as debris discs, where the dust and gas is inferred to be secondary, created in the destruction of planetesimals that must be volatile-rich to replenish the gas (Wyatt 2008; Dent et al. 2014; Marino et al. 2016; Moór et al. 2017; Matrà et al. 2017; Kral et al. 2017). The transition between the two types of disc is not well understood, but is thought to involve a combination of gas being accreted onto the star, or being expelled from the system by disc winds driven by photoevaporation or magneto-hydrodynamics (MHD), as well as planet formation processes (Williams \& Cieza 2011; Wyatt et al. 2015; Lesur 2020). Class III stars are those in star forming regions for which infrared emission shows an absence of hot dust suggesting that the star's protoplanetary disc has either recently dispersed or is in the process of dispersal (Adams et al.

^ E-mail: j1638@cam.ac.uk
1987). However, these stars usually have limited constraints on the presence of cold dust or gas with which to constrain their nature.

A recent ALMA survey of class III stars in Lupus found dust in several systems with dust masses orders of magnitude lower than protoplanetary disc levels and consistent with originating in the progenitors of debris discs seen at later ages, though this does not rule out the possibility that this dust is a remnant of the protoplanetary disc (Lovell et al. 2020). For one of these class III stars, NO Lup, J = 3-2 CO gas was also detected, allowing a more thorough assessment of the nature of its circumstellar environment. Comparison of the inferred mass of $\mathrm{CO}$ with that of the $10 \mathrm{Myr}$ old M star TWA 7, for which the level was consistent with secondary production (i.e., $0.8-80 \times 10^{-6} M_{\oplus}$, see Matrà et al. 2019), shows that the $\mathrm{CO}$ could indeed be secondary, though as for the dust interpretation, such plausibility does not preclude the possibility that the $\mathrm{CO}$ is primordial.

This paper presents a detailed analysis of the CO detected towards NO Lup, using spectral and spatial data not reported in Lovell et al. (2020), constraining the kinematic structure of the 
gas disc. While circumstellar gas is usually seen in a Keplerian disc, other gas morphologies are possible, such as an outflowing component which could be a feature of either a depleting primordial disc (Haworth \& Owen 2020) or a debris disc. In \$2 we provide a summary of the NO Lup system, and introduce new X-Shooter observations. In $\S 3$ we extend the analysis of the $\mathrm{CO}$ gas by showing the problem with modelling this as a Keplerian disc, and show better consistency in $\$ 4$ by modelling this with an outward radial velocity. We interpret our results in $\S 5$, and conclude in $\S 6$.

\section{NO LUP IN CONTEXT}

NO Lup (2MASS J160311.8-323920) is located in Cloud I of Lupus at $\alpha=16: 03: 11.812, \delta=-32: 39: 20.31$ (J2000) at a distance of $133.7 \pm 0.7$ pc (Gaia Collaboration et al. 2018). NO Lup has previously been classified spectrally as non-accreting (Cieza et al. 2013), and as a K7 star (Krautter et al. 1997). The latter is consistent with its Gaia DR2 temperature, $T_{\mathrm{eff}}=3994 \mathrm{~K}$, and stellar luminosity, $L_{\star}=0.287 L_{\odot}$ and its well constrained spectral energy distribution (given observations with WISE, Spitzer, and 2MASS, which found the blackbody planetesimal belt radius as $R_{\mathrm{BB}}=3.2 \pm 0.3 \mathrm{au}$, see Lovell et al. 2020). Analysing the X-Shooter spectrum of NO Lup (project 093.C-0506 A), we confirm both spectral and accretion analyses. By reducing this data using the standard pipeline version 3.5.0 (Modigliani et al. 2010), we show in Fig. 1 (top and middle) the full and zoomed-in X-Shooter spectra for NO Lup, which are consistent with the spectral features of the well characterised class III K7 star, SO879. In the lower panel we show the $\mathrm{H} \alpha$ emission line with an $\mathrm{EW}=-2.80 \pm 0.15 \AA$, which we find to be centred on a radial velocity (RV) of $-3.5 \pm 2.0 \mathrm{~km} \mathrm{~s}^{-1}$, consistent with the Gaia DR2 RV of $-1.93 \pm 4.08 \mathrm{~km} \mathrm{~s}^{-1}$, and the average RV of Lupus stars, $\mathrm{RV}_{\text {Lup }}=2.8 \pm 4.2 \mathrm{~km} \mathrm{~s}^{-1}$ (Frasca et al. 2017). This EW is consistent with non-accretion and only slightly higher than, but also consistent with, the line width of non-accreting SO879. Hardy et al. (2015) estimated the stellar mass of NO Lup to be $M_{\star}=0.7 M_{\odot}$. With the models of Siess et al. (2000) and Baraffe et al. (2015), and the Gaia DR2 $L_{\star}$ and $\mathrm{T}_{\text {eff }}$, we estimated $M_{\star}$ between $0.7-0.8 M_{\odot}$, consistent with the literature. Although NO Lup has significant emission above the photosphere at 12 and $24 \mu \mathrm{m}$, these excesses are small, resulting in mid-IR spectral slopes steeper than those of protoplanetary discs. Unresolved continuum emission was detected by $A L M A$, implying a dust mass of $0.036 \pm 0.007 M_{\oplus}$ and disc radius $<56$ au (Lovell et al. 2020). These observations also detect CO J=3-2 line emission, with $F_{\mathrm{CO}}=0.29 \pm 0.07 \mathrm{Jy} \mathrm{km} \mathrm{s}^{-1}$, and a width of $\sim 19 \mathrm{~km} \mathrm{~s}^{-1}$ (between -11.0 to $+8.1 \mathrm{~km} \mathrm{~s}^{-1}$ ), consistent with being centred on the stellar RV discussed above. Assuming this emission is optically thin and in LTE at $T=50 K$, this flux corresponds to a $\mathrm{CO}$ gas mass of $4.9 \pm 1.1 \times 10^{-5} M_{\oplus}$, which for an ISM CO abundance implies a gas-to-dust ratio of $1.0 \pm 0.4$ (Lovell et al. 2020). We note that a wider temperature range of $20-100 \mathrm{~K}$, consistent with the range of literature gas temperatures, could change this gas mass by at most a factor of $\sim 2$ (see equations 2 and 8 of Matrà et al. 2017).

\section{THE PROBLEM WITH A KEPLERIAN DISC}

Assuming the gas is in Keplerian rotation, the width of the $\mathrm{CO}$ line can be used to estimate the spatial extent of the emission. For example, if the star has a mass $\sim 0.7 M_{\odot}$ (see $\S 2$ ), the line width indicates a radius of $\sim 10$ au (or smaller if the disc is not edge on), well below the spatial resolution limit of our measurements. However, we find

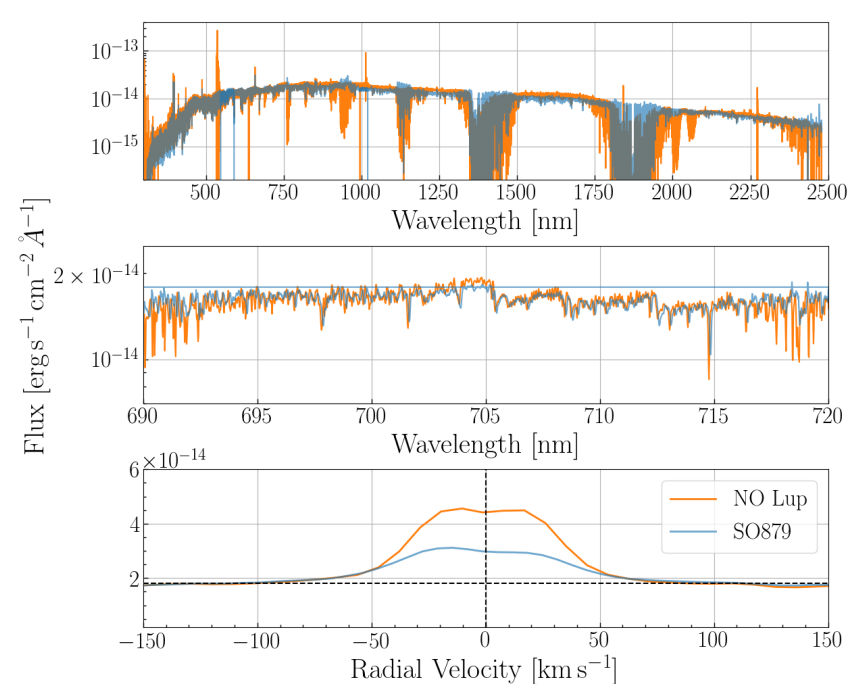

Figure 1. X-Shooter spectra for NO Lup and SO879. Top: full X-Shooter range. Middle: zoomed-in 690-720 nm range. Bottom: H $\alpha$ line. SO879's flux is scaled to NO Lup's median source flux between $698-702 \mathrm{~nm}$, and is wavelength corrected to have a common RV with NO Lup based on SO879's DR2 RV of $30.10 \pm 0.21 \mathrm{~km} \mathrm{~s}^{-1}$ (Gaia Collaboration et al. 2018).

that the $\mathrm{CO}$ emission is spatially resolved. Fig. 2 shows that the centroid of the $\mathrm{CO}$ emission transitions from the South-West to the North-East when binned in channels with decreasing RV, as expected for emission that originates in a disc inclined to the plane of the sky. To resolve this emission given a beam of $(0.94 \times 0.82)^{\prime \prime}$ (at position angle $70^{\circ}$ ), the disc emission would have to have a radius $\gtrsim 50 \mathrm{au}$. This however would then be inconsistent with the previously inferred $\sim 10$ au radius for the assumed stellar mass. This conclusion can also be illustrated with the position-velocity (PV) diagram in Fig. 3 (see left-hand plot 1), produced with a $36^{\circ}$ position angle and $2^{\prime \prime}$ slit width, which shows how the offset varies with radial velocity. This agrees with the previous analysis that the velocities are unexpectedly high at a large separation (nearly $10 \mathrm{~km} \mathrm{~s}^{-1}$ at $\sim 0.5^{\prime \prime}=66 \mathrm{au}$ ). The curves and radial extension line on this same plot show that for gas to be in a Keplerian orbit the stellar mass would have to be $\sim 11 M_{\odot}$, i.e., much higher than $0.7 M_{\odot}$.

We use a simple model to explore this Keplerian disc interpretation by assuming the gas is in a Keplerian orbit with the stellar mass of NO Lup left as a free parameter, $M_{\star}$. This Disc Model computes the density of emitting $\mathrm{CO}$ for each pixel in a cube that has RA, Dec, and line of sight, $z$, as axes. The orientation, position and RV of the disc in the cube are set by the ascending node, $\Omega$ (i.e., the PA of the disc major axis), the inclination, $i$, a phase centre offset in RA and Dec ( $\mathrm{x}_{\text {off }}$ and $\left.\mathrm{y}_{\text {off }}\right)$, the systemic velocity, $v_{\text {sys }}$, and the fixed distance to NO Lup of $133.7 \mathrm{pc}$ (see §2). The model assumes that the $\mathrm{CO}$ density can be modelled with a total flux, $F$, and a power-law radial profile, defined between $r_{\text {in }}$ and $r_{\text {out }}$ with index $p$, for which the volume density goes as $r^{p-1}$. The model assumes a Gaussian scale height with a fixed aspect ratio, $\sigma_{h}=0.05$, meaning that the surface density goes as $\Sigma \propto r^{p}$. We fitted the visibilities of channels -15.1 to $+6.9 \mathrm{~km} \mathrm{~s}^{-1}$ from the CO line (as done in Kennedy et al. 2019), using the emcee package (Foreman-Mackey et al. 2013), with 40 walkers and 2000 steps to achieve convergence. The fit gives reasonable results, showing no residuals in the PV diagram $>3 \sigma$ (see Fig. 3, plots 2 and 3), with the best-fit model values shown in Table 1 . We note that the 


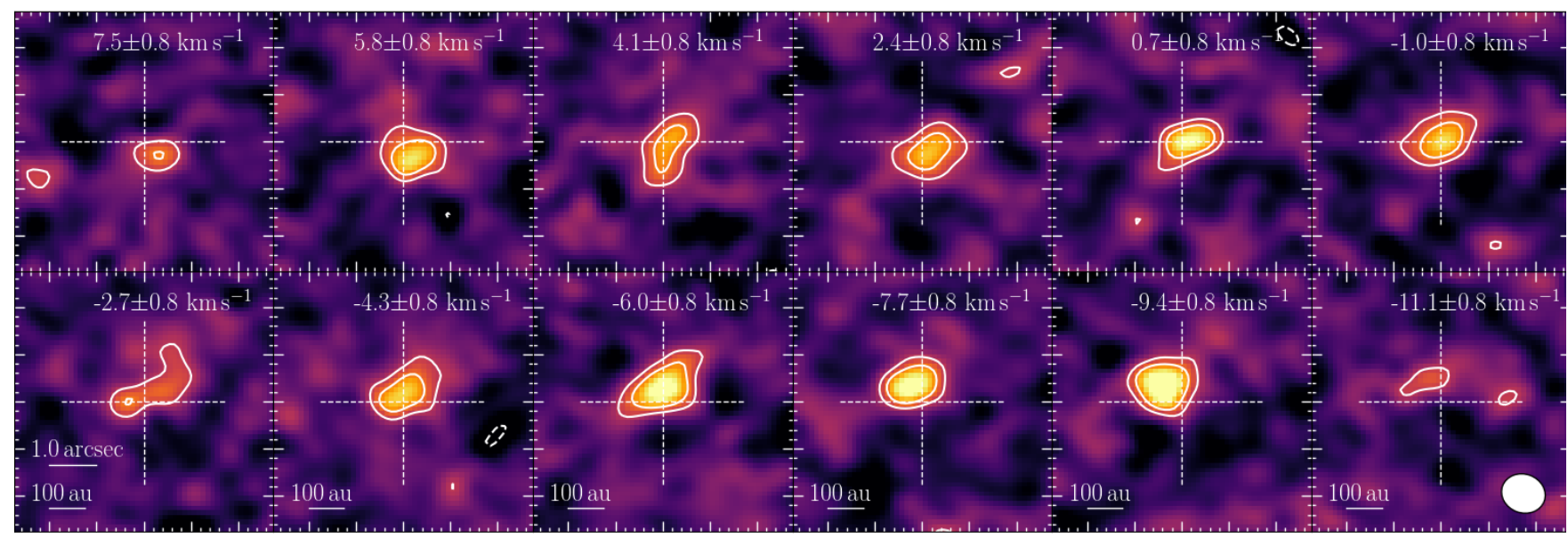

Figure 2. Averaged channel map $\left(4 \sim 0.4 \mathrm{~km} \mathrm{~s}^{-1}\right.$ channels per plot), showing the \pm 3 and $5 \sigma$ emission significance. In all plots North is up, East is left, contour lines represent \pm 3 and $\pm 5 \sigma, 100$ au scale bars are shown in the lower-left of the bottom panel plots, the synthesised beam is shown in the bottom-right plot, and the major axis ticks are $1^{\prime \prime}$ wide.

Table 1. Best-fit model parameters. D: Disc Model, and O: Outflow Model.

\begin{tabular}{c|c|c|c|c|c|c}
\hline \hline Mod & $\begin{array}{c}\Omega \\
\mathrm{deg}\end{array}$ & $\begin{array}{c}i \\
\mathrm{deg}\end{array}$ & $\begin{array}{c}r_{\text {in }} \\
\mathrm{au}\end{array}$ & $\begin{array}{c}r_{\text {out }} \\
\mathrm{au}\end{array}$ & $\begin{array}{c}M_{\star} \\
M_{\odot}\end{array}$ & $\begin{array}{c}v_{r} \\
\mathrm{~km} \mathrm{~s}^{-1}\end{array}$ \\
\hline $\mathrm{D}$ & $36 \pm 3$ & $42 \pm 5$ & $35 \pm 8$ & $90 \pm 5$ & $11.1 \pm 1.9$ & - \\
$\mathrm{O}$ & $115 \pm 3$ & $20 \pm 4$ & $20 \pm 4$ & $120 \pm 14$ & $0.70 \pm 0.05$ & $22 \pm 4$ \\
\hline
\end{tabular}

best-fit $v_{\text {sys }}=-3.8 \mathrm{~km} \mathrm{~s}^{-1}$ is consistent with that in $\S 2$, and that this model did not constrain $p$, which uniformly varied between the imposed limits of -2 to 1 . A similar result was found with a Gaussian radial distribution. This fitting procedure therefore comes to the same conclusion as shown earlier in requiring a stellar mass $M_{\star}=11.1 \pm 1.9 M_{\odot}$ that is significantly greater than expected. Since the stellar luminosity rules out $\sim 10 \mathrm{~K}$-type stars within 10 s of au of NO Lup, and the X-Shooter spectra rules out NO Lup being a mis-classified star, we conclude that we cannot consistently interpret the $\mathrm{CO}$ emission as originating from a Keplerian disc.

\section{AN OUTFLOWING GAS INTERPRETATION}

Having ruled out the Keplerian disc interpretation, we explore the possibility that the gas emission is dominated by a radially outflowing velocity component. We define an Outflow Model with a stellar mass fixed at $0.7 M_{\odot}$ (which sets the azimuthal velocity), where the $\mathrm{CO}$ emission extends between $r_{\text {in }}$ and $r_{\text {out }}$ with a radial velocity, $v_{r}$, which models the CO gas outflow. This is a variant of the Disc Model of $\S 3$, with the same disc parameters and geometry, except that the gas also has an additional radial velocity component and the vertical density distribution is modelled as uniform, with fixed lower and upper edges, such that a vertical cross-section through the disc looks like a wedge with an opening angle $\delta h$. Allowing for a larger scale height is a rough approximation to disc wind models, where material flows both vertically off the disc and radially outwards. We fitted the visibilities as described above for the Disc Model. We again find reasonable results (see Fig. 3, plots 4 and 5 ), and quote the best-fit model parameters in Table 1, also finding $v_{\text {sys }}=-3.8 \mathrm{~km} \mathrm{~s}^{-1}$, and an unconstrained $p$, which we therefore fixed as $p=-1^{1}$. The Outflow Model finds $v_{r} \approx 22 \mathrm{~km} \mathrm{~s}^{-1}$, which is higher than the measured velocity from the $\mathrm{CO}$ line width and thus dominates the azimuthal velocity for the modelled $r_{\text {in }}$ and $r_{\text {out }}$. The Outflow Model finds a large opening angle of $\delta h=0.3 \pm 0.1$; the disc inclination is required to be low to reproduce the spatial extent, thus the scale height is large to maximise the radial component of the velocity, and $v_{r}$ is significantly larger than the minimum possible value of $\sim 19 / 2 \mathrm{~km} \mathrm{~s}^{-1}$. Though the flow in the model is mostly radial, the large scale height can be thought of as approximating the significant vertical component present in outflow models. Despite the simplicity of assuming the gas to have an additional radial velocity component, this model shows that the observations are broadly consistent with a scenario in which the gas is radially outflowing.

\section{FIRST IMAGED CLASS III GAS DISPERSAL}

Whilst we model the CO emission over all the channels in which it is detected, for simplicity we have plotted the two models of $\$ 3$ and 4 in Fig. 3 as PV diagrams, with their corresponding moment- 0 maps as thumbnails. These show that whereas the models have similar PV diagrams, the position angles of their disc midplanes are $\sim 90^{\circ}$ different, discussed further in Appendix A. At low spatial resolution, Keplerian disc models and high radial velocity outflowing models (i.e., those in which the radial velocity dominates the azimuthal velocity) can have indistinguishable PV diagrams. This degeneracy can be broken with higher resolution imaging to measure the PA of the continuum emission which would confirm the radial (or azimuthal) nature of observed $\mathrm{CO}$ velocities.

The simple Outflow Model presented in $\$ 4$ showed that the observations can be fitted with a constant $\sim 22 \mathrm{~km} \mathrm{~s}^{-1}$ radial velocity component. While it may be possible to explain the observations with a lower outflow velocity, for example with a more detailed model of the gas kinematics, any gas flow must be $\gtrsim 10 \mathrm{~km} \mathrm{~s}^{-1}$, given the spectrum line width of $\sim 19 \mathrm{~km} \mathrm{~s}^{-1}$. This is high compared to the photoevaporative models of Haworth \& Owen (2020), in which the outflowing wind velocity is $\sim 3 \mathrm{~km} \mathrm{~s}^{-1}$.

\footnotetext{
${ }^{1} p=-1$ is the value expected for mass conservation if the $\mathrm{CO}$ is being created at the inner disc edge.
} 

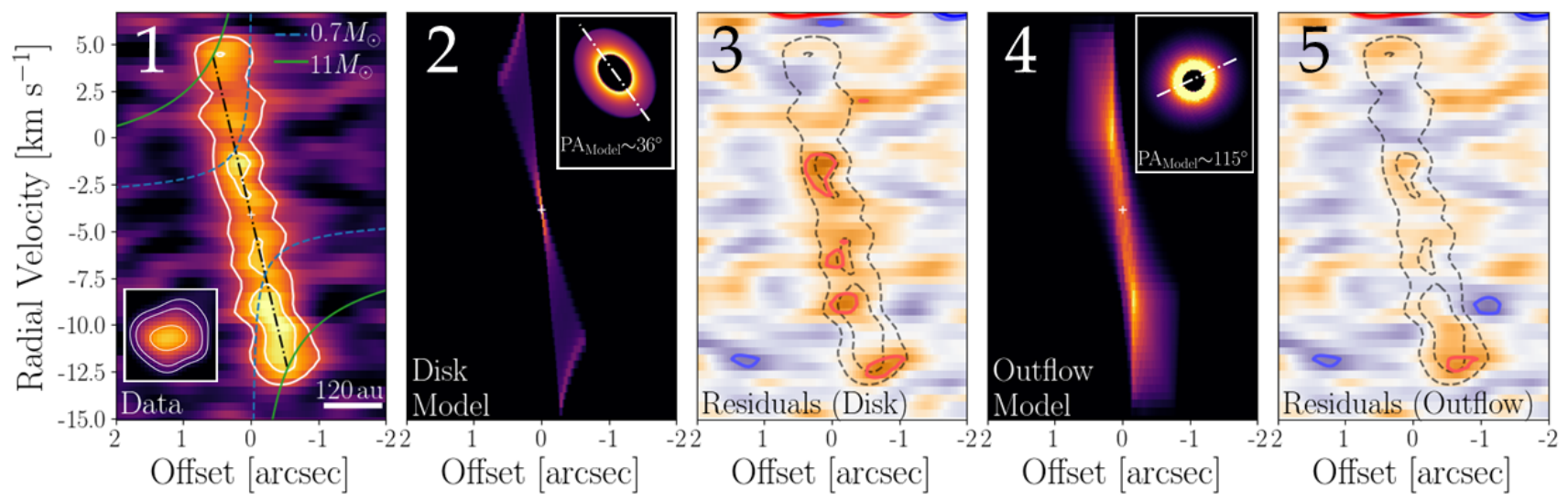

Figure 3. Plot 1: PV diagram showing the distribution of velocities present at different offsets along a slit width of $2^{\prime \prime}$ and position angle of $36^{\circ}$. The contours show 3 and $5 \sigma$ emission. The curves demonstrate the maximum allowed radial velocities for Keplerian motion around a $11 M_{\odot}$ star (green solid) and a $0.7 M_{\odot}$ star (blue dashed), and the radial velocity expected for a disc with an 80 au radius (black dot-dash slope). The lower-left thumbnail shows the Moment- 0 map (box extent $\sim 300$ au, with 3,6, and $9 \sigma$ contours). Plots 2 and 4: Model PV diagrams using wider slits covering the full model extent, however we note that the emission beyond $2^{\prime \prime}$ is negligible. The thumbnails show the model Moment-0 maps ( 300 au box widths), and PAs (see Table 1). Plots 3 and 5: Residual PV diagrams, with \pm 2 and $\pm 3 \sigma$ residuals shown in blue/orange, and the same contours as the data (black dashed).

In fact, this high velocity rules out a scenario in which the gas is a pure photoevaporative wind, since such a wind velocity should be set by the sound speed, and so to reach even $\sim 10 \mathrm{~km} \mathrm{~s}^{-1}$ would require the $\mathrm{CO}$ gas to be at $T \sim 10,000 \mathrm{~K}$, which exceeds the $\mathrm{CO}$ thermal dissociation temperature. To accelerate $\mathrm{CO}$ gas to such high speeds, additional forces are required (e.g., such as MHD driven winds, see Lesur 2020, and references therein). Winds thought to be driven by the disc magnetic field have been observed towards less evolved class II stars with similarly high velocities (see Pontoppidan et al. 2011). Theoretically, magnetically driven disc winds can be launched with velocities $\sim$ several factors of the Keplerian velocity at $10 \mathrm{~s}$ of au (Bai 2016), which at the location of our disc inner edge $\left(r_{\mathrm{in}}=20 \pm 4 \mathrm{au}\right)$ is consistent with our modelled outflow velocity. Thus the $10 \mathrm{~s}$ of $\mathrm{km} \mathrm{s}^{-1}$ velocities inferred here are plausible via this mechanism. Though we are not aware of theoretical works that have looked into the launching of a magnetised disc wind in the conditions of a debris disc, we note that $4-12 \mathrm{~km} \mathrm{~s}^{-1}$ dust outflows were detected towards AU Mic by Boccaletti et al. (2018), and it is worth exploring whether these are launched by a similar mechanism. Interpretation of NO Lup's CO emission may therefore require modelling a $3 \mathrm{D}$ velocity field and MHD driving, in addition to $\mathrm{CO}$ photodissociation and shielding (which we discuss below, Kral et al. 2019; Marino et al. 2020).

While we have discussed the parameter $v_{r}$ in the Outflow Model as an outflowing velocity, the fit to the observations is insensitive to the sign of $v_{r}$, thus we next explore whether the gas is more likely to be infalling or outflowing. To do so, we compare the observed $\mathrm{CO}$ mass loss rate with the upper limit on the $\mathrm{CO}$ accretion rate. Assuming $\dot{M}_{\mathrm{CO}}=M_{\mathrm{CO}} \cdot v_{r} / R$, we find $\dot{M}_{\mathrm{CO}} \sim 3 M_{\oplus} \mathrm{Myr}^{-1}$, for $M_{\mathrm{CO}} \sim 5 \times 10^{-5} M_{\oplus}$, at $v_{r} \sim 22 \mathrm{~km} \mathrm{~s}^{-1}$, from the mean disc radius $R=\left(r_{\text {in }}+r_{\text {out }}\right) / 2 \sim 70$ au. Since the estimate of $M_{\mathrm{CO}}$ assumes it is in LTE and that the gas is optically thin, this mass loss rate is a lower limit. Next, by fitting a polynomial to the continuum near the $\mathrm{H} \alpha$ line (with the EW stated in \$2), we obtain a continuum-subtracted line flux of $\sim 2.9 \times 10^{-15} \mathrm{erg} \mathrm{s}^{-1} \mathrm{~cm}^{-2}$, from which we estimate the accretion luminosity as $\log L_{\text {acc }} / L_{\odot} \sim-3.04$, using the empirical relation between line luminosity and accretion luminosity of Alcalá et al. (2017). With $L_{\text {acc }}, M_{\star}$ and $L_{\star}$ from $\S 2$, and a stellar radius of $R_{\star} \sim 1.3 R_{\odot}$ (Gaia Collaboration et al. 2018), we find a $3 \sigma$ upper limit $\mathrm{CO}$ mass accretion rate of $<0.1 M_{\oplus} \mathrm{Myr}^{-1}$, for an ISM $\mathrm{H}_{2} / \mathrm{CO}$ abundance ratio of 10,000 (i.e., consistent with the ratio in primordial gas). This is consistent with other non-accreting class III stars (see Manara et al. 2013), however it is more than 30 times lower than the inferred CO mass loss rate, indicating that if primordial the $\mathrm{CO}$ gas cannot be inflowing. While this cannot rule out inflowing gas for lower $\mathrm{H}_{2} / \mathrm{CO}$ ratios (e.g., if the gas is produced in a secondary scenario), we rule out inflowing secondary gas later. However, the upper limit on the CO mass accretion rate may provide an important constraint on models for the outflow that require an inflowing component to conserve angular momentum.

To explore the origin of the gas, we first note that $\mathrm{CO}$ at $10-20 \mathrm{~km} \mathrm{~s}^{-1}$ (i.e., $2-4 \mathrm{au} \mathrm{yr}^{-1}$ ) would travel $\gtrsim 200 \mathrm{au}$ in $100 \mathrm{yr}$, i.e., over the CO photodissociation time (Visser et al. 2009), although if well shielded the $\mathrm{CO}$ could survive much longer. This may suggest that the gas was formed during the recent break-up of a massive planetesimal, however such an event would be both rare and leave a distinctive asymmetry in the gas distribution (Jackson et al. 2014) which we do not observe. Rather, the measured $\sim 130$ au extent of the $\mathrm{CO}$ gas could still be consistent with a $>100 \mathrm{yr}$ lifetime, as at larger distances from the star cooler CO is less collisionally excited, and so difficult to detect (Matrà et al. 2015). Thus, comparing the extent, velocity and gas lifetime does not lead to strong constraints, though this may not be the case for neutral CI gas, which is likely a better probe of outflowing gas at larger distances, as suggested by Haworth \& Owen (2020). Given typical stellar/disc timescales of 0.1-1 Myr, the high velocity however suggests that the $\mathrm{CO}$ must be continuously replenished, as it is extremely unlikely that the $\mathrm{CO}$ was imaged within $100 \mathrm{yr}$ after a single or final production event.

The $\mathrm{CO}$ reservoir that replenishes the gas may either be in gaseous form (a protoplanetary disc remnant) or in solid form (icy planetesimals in a debris disc). Protoplanetary discs are expected to disperse on $\sim 100 \mathrm{kyr}$ timescales (e.g. Ercolano \& Pascucci 2017). While NO Lup's SED suggests that it has already lost its protoplanetary disc, the dust may have dispersed first, leaving a 
primordial CO reservoir (e.g., as suggested by Owen \& Kollmeier 2019). The detection of CO gas towards $1 / 30$ of the $2 \mathrm{Myr}$ old Lupus class III stars in Lovell et al. (2020) implies plausible dispersal timescales for such primordial gas remnants of $\sim 70 \mathrm{kyr}$. A potential problem with this, however, is that no $\mathrm{CO}$ reservoir with a Keplerian disc signature is present in our observations. For such a $\mathrm{CO}$ reservoir to go undetected its surface brightness, which in the optically thick limit is $I_{v}=B_{v}(T) A / A_{\text {beam }}$ (where $A$ is the $\mathrm{CO}$ emission area, and $A_{\text {beam }}$ is the beam area), should be below the $3 \sigma$ noise level of $\sim 9$ mJy beam $^{-1}$ (see Lovell et al. 2020). If CO emission fills the beam $\left(A=A_{\text {beam }}\right)$, then this upper limit implies a temperature below $\sim 4 \mathrm{~K}$, significantly below the $\mathrm{CO}$ sublimation temperature and thus unlikely. For a more reasonable temperature of $50 \mathrm{~K}$, the $\mathrm{CO}$ emitting area would need to be $\sim 150$ times smaller than the beam, and thus a ring at $r_{\text {in }} \sim 20$ au must have a width narrower than $0.01 \mathrm{au}$, which is also unlikely. This argues against an optically thick ring of $\mathrm{CO}$ being the gas source, though further high-resolution imaging is required to definitively conclude this. A more plausible explanation may be that the wind is replenished by a reservoir of $\mathrm{CO}$ in icy planetesimals in the $R_{\mathrm{BB}} \sim 3$ au belt. Since blackbody disc radii estimates can be $\gtrsim 5 \times$ smaller than physical planetesimal belt radii (see Eq.8 of Pawellek \& Krivov 2015), this $R_{\mathrm{BB}}$ is consistent with the modelled inner edge of the gas. Moreover, if the $\mathrm{CO}$ gas in this scenario is produced in the known planetesimal belt and observed at $\sim 130$ au, this backs up the previous claim that the gas must be outflowing. Thus, we may be witnessing a short-lived phase after protoplanetary disc dispersal in which CO ice is released and dispersed. For example, it may be that following primordial gas dispersal a previously stable reservoir of $\mathrm{CO}$ ice became susceptible to sublimation (similar to the mechanism suggested on 486958 Arrokoth by Steckloff et al. 2020), or CO was released as planetesimals were ground down in collisions (Marino et al. 2020). If this is the case, then we may find more examples of class III stars with rapidly dispersing gas winds. Gas winds have not been seen towards older gaseous debris discs which may indicate that these winds are linked to the evolutionary stage (or spectral type) of the star, or perhaps suppressed by the build-up of other gaseous species over many Myr.

\section{CONCLUSIONS}

By analysing the $\mathrm{CO}$ gas detected towards the class III star, NO Lup, we have demonstrated that the $\mathrm{CO}$ has a high velocity width and is spatially resolved. Although we showed that this can be fitted with a Keplerian Disc Model, this requires the stellar mass of NO Lup to be implausibly high, i.e., 10 times higher than expected. Instead, we have shown that the gas may be outflowing with a high radial velocity, explaining the $\sim 19 \mathrm{~km} \mathrm{~s}^{-1}$ width and $\sim 130$ au spatial scale. We conclude that this gas is outflowing and is being continually replenished, and suggest possible interpretations. We note further work to explore the nature of this detection, such as new high resolution imaging of the continuum, measurements for the neutral CI gas, and detailed modelling.

\section{ACKNOWLEDGEMENTS}

We thank the anonymous reviewer for their comments which improved the quality of this work. JBL is supported by an STFC postgraduate studentship. GMK is supported by the Royal Society as a Royal Society University Research Fellow. SM is supported by a
Research Fellowship from Jesus College, Cambridge. GR acknowledges support from the Netherlands Organisation for Scientific Research (NWO, program number 016.Veni.192.233). M.T. has been supported by the UK Science and Technology research Council (STFC), and by the European Union's Horizon 2020 research and innovation programme under the Marie Sklodowska-Curie grant agreement No. 823823 (RISE DUSTBUSTERS project). JPW acknowledges support from NSF grant AST-1907486. MK gratefully acknowledges funding by the University of Tartu ASTRA project 2014-2020.4.01.16-0029 KOMEET. This work was partly supported by the Deutsche Forschungs-Gemeinschaft (DFG, German Research Foundation) - Ref no. FOR 2634/1 TE 1024/1-1. This work was partly supported by the Italian Ministero dell'Istruzione, Università e Ricerca (MIUR) through the grant Progetti Premiali 2012 iALMA (CUP C52I13000140001) and by the DFG cluster of excellence Origin and Structure of the Universe (www.universecluster.de).

\section{DATA AVAILABILITY}

This work makes use of the following ALMA data: ADS/ JAO.ALMA \#2018.1.00437.S. ALMA is a partnership of ESO (representing its member states), NSF (USA) and NINS (Japan), together with NRC (Canada), MOST and ASIAA (Taiwan), and KASI (Republic of Korea), in cooperation with the Republic of Chile. The Joint ALMA Observatory is operated by ESO, AUI/NRAO and NAOJ. Based on observations collected at the European Southern Observatory under ESO programme 093.C-0506(A).

\section{REFERENCES}

Adams F. C., Lada C. J., Shu F. H., 1987, ApJ, 312, 788

Alcalá J. M., et al., 2017, A\&A, 600, A20

Bai X.-N., 2016, ApJ, 821, 80

Baraffe I., Homeier D., Allard F., Chabrier G., 2015, A\&A, 577, A42

Boccaletti A., et al., 2018, A\&A, 614, A52

Cieza L. A., et al., 2013, ApJ, 762, 100

Dent W. R. F., et al., 2014, Science, 343, 1490

Ercolano B., Pascucci I., 2017, Royal Society Open Science, 4, 170114

Foreman-Mackey D., Hogg D. W., Lang D., Goodman J., 2013, Publications of the Astronomical Society of the Pacific, 125, 306

Frasca A., Biazzo K., Alcalá J. M., Manara C. F., Stelzer B., Covino E., Antoniucci S., 2017, A\&A, 602, A33

Gaia Collaboration et al., 2018, Astronomy and Astrophysics, 616, A1

Hardy A., et al., 2015, A\&A, 583, A66

Haworth T. J., Owen J. E., 2020, MNRAS, 492, 5030

Jackson A. P., Wyatt M. C., Bonsor A., Veras D., 2014, MNRAS, 440, 3757

Kennedy G. M., et al., 2019, Nature Astronomy, 3, 230

Kral Q., Matrà L., Wyatt M. C., Kennedy G. M., 2017, Monthly Notices of the Astronomical Society, 469, 521

Kral Q., Marino S., Wyatt M. C., Kama M., Matrà L., 2019, MNRAS, 489, 3670

Krautter J., Wichmann R., Schmitt J. H. M. M., Alcala J. M., Neuhauser R., Terranegra L., 1997, Astronomy and Astrophysics Supplement Series, 123,329

Lesur G., 2020, arXiv e-prints, p. arXiv:2007.15967

Lovell J. B., et al., 2020, Monthly Notices of the Royal Astronomical Society

Manara C. F., et al., 2013, A\&A, 551, A107

Marino S., et al., 2016, Monthly Notices of the Royal Astronomical Society, 460, 2933

Marino S., Flock M., Henning T., Kral Q., Matrà L., Wyatt M. C., 2020, MNRAS, 492, 4409 


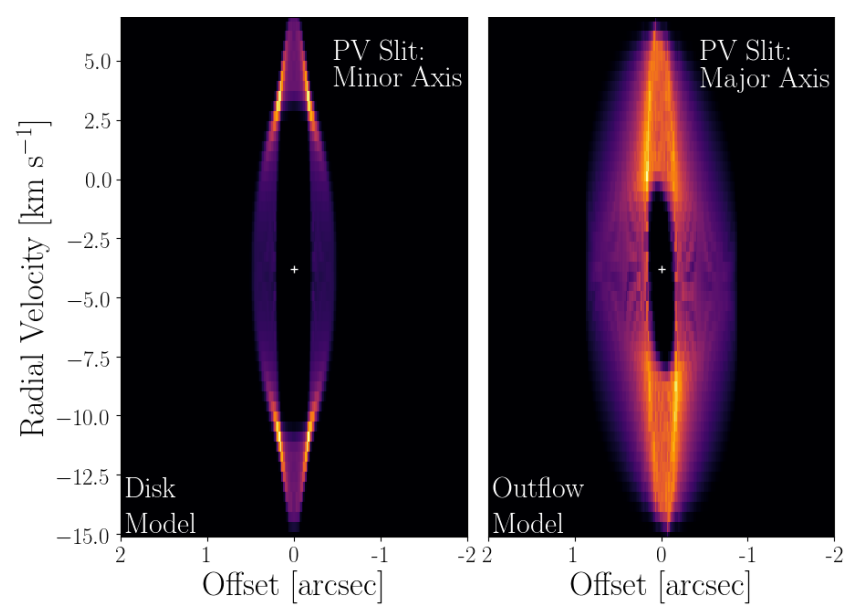

Figure A1. PV diagrams for the Disc Model and Outflow Model with their slits taken at $90^{\circ}$ to those in Fig. 3 to demonstrate their elliptical nature in this orientation. The Outflow Model is rotated slightly anti-clockwise by the Keplerian motion, though this would be much greater if the radial and Keplerian velocities were more similar.

Matrà L., Panić O., Wyatt M. C., Dent W. R. F., 2015, Monthly Notices of the Royal Astronomical Society, 447, 3936

Matrà L., et al., 2017, Monthly Notices of the Royal Astronomical Society, 464,1415

Matrà L., Öberg K. I., Wilner D. J., Olofsson J., Bayo A., 2019, AJ, 157, 117

Modigliani A., et al., 2010, in Observatory Operations: Strategies, Processes, and Systems III. p. 773728, doi:10.1117/12.857211

Moór A., et al., 2017, ApJ, 849, 123

Owen J. E., Kollmeier J. A., 2019, MNRAS, 487, 3702

Pawellek N., Krivov A. V., 2015, Monthly Notices of the Royal Astronomical Society, 454, 3207

Pontoppidan K. M., Blake G. A., Smette A., 2011, ApJ, 733, 84

Siess L., Dufour E., Forestini M., 2000, A\&A, 358, 593

Steckloff J. K., Lisse C. M., Safrit T. K., Bosh A. S., Lyra W., Sarid G., 2020, arXiv e-prints, p. arXiv:2007.12657

Visser R., van Dishoeck E. F., Black J. H., 2009, A\&A, 503, 323

Williams J. P., Cieza L. A., 2011, ARA\&A, 49, 67

Wyatt M. C., 2008, Annual Review of Astronomy and Astrophysics, 46, 339

Wyatt M. C., Panić O., Kennedy G. M., Matrà L., 2015, Ap\&SS, 357, 103

\section{APPENDIX A: PV DIAGRAMS}

In Table 1 we show that the two models that fit the observations have disc position angles that are $\sim 90^{\circ}$ different. Since our modelling fits all channel maps in the data set (i.e., it does not fit a PV diagram) we have a choice in how we present this. In Fig. 3 we used a slit angle of $36^{\circ}$, i.e., parallel to direction of motion of the peak in the channel maps, the Disc Model major axis, and the Outflow Model minor axis, which produced a stripe (see panels 1, 2 and 4). If instead we place the PV diagram slit PA along the major axis of the Outflow Model and the minor axis of the Disc Model, these yield model PV diagrams that are then elliptical as can be seen in Fig. A1, and similar to those presented in Haworth \& Owen (2020). Thus, although the choice of slit PA does not bias our modelling, a different choice will result in a different visualisation, but not one that can distinguish between the Keplerian Disc Model and the Outflow Model.

This paper has been typeset from a $\mathrm{T}_{\mathrm{E}} \mathrm{X} / \mathrm{L} \mathrm{T} \mathrm{T}_{\mathrm{E}} \mathrm{X}$ file prepared by the author. 